\title{
ENTIRE FUNCTIONS OF BOUNDED INDEX
}

S. M. SHAH

1. Introduction. A polynomial is of bounded index. ${ }^{1}$ In this paper we show that an entire function of bounded index is of exponential type. Furthermore we show that there exist entire functions of unbounded index and arbitrarily slow growth. If an entire function satisfies a linear differential equation with constant coefficients, then it is of bounded index. We prove

THEOREM 1. If $f(z)$ is an entire function of bounded index, then

$$
\limsup _{r \rightarrow \infty} \frac{\log M(r, f)}{r} \leqq N+1,
$$

where $N$ is the index of $f$. This result is sharp.

TheOREM 2. Given any function $\phi(r)$ such that $\phi(r) \geqq c>0$ for $r \geqq 0$ and $\log \phi(r) / \log r \rightarrow \infty$ as $r \rightarrow \infty$, there exists an entire function $f(z)$ of unbounded index, such that for every $r \geqq 0, M(r, f) \leqq \phi(r)$.

THEOREM 3. If $f(z)$ is a transcendental entire function and satisfies the linear differential equation

$$
f^{(n)}+a_{1} f^{(n-1)}+\cdots+a_{n} f=0,
$$

of order $n$, with constant coefficients, then $f(z)$ is of bounded index. Furthermore, the index $N \leqq p$ where $p$ is any integer such that $p \geqq n-1$ and

$$
\frac{\left|a_{1}\right|}{p+1}+\frac{\left|a_{2}\right|}{p(p+1)}+\cdots+\frac{\left|a_{n}\right|}{(p-(n-2)) \cdots(p+1)} \leqq 1 .
$$

COROLLARY. If $p$ is any integer such that

$$
p \geqq n-2+\left|a_{1}\right|+\left|a_{2}\right|^{1 / 2}+\cdots+\left|a_{n}\right|^{1 / n},
$$

then $N \leqq p$.

Remarks. (i) The hypothesis in Theorem 3 implies that $n \geqq 1$ and $\max \left(\left|a_{1}\right|, \cdots,\left|a_{n}\right|\right)>0$.

(ii) A theorem similar to Theorem 2 is given by Lepson [2]. Our method of construction is different from that of Lepson.

2. Proof of Theorem 1. We require the following lemmas.

Presented to the Society, April 19, 1967 ; received by the editors May 9, 1967.

${ }^{1}$ For definition, see the paper by Fred Gross, Entire functions of bounded index, Proc. Amer. Math. Soc. 18 (1967), 974-980. See also [2]. 
Lemma 1 [3]. Let $F(r)$ be positive and nondecreasing for $r>r_{0}$, and suppose that

$$
\limsup _{r \rightarrow \infty} \frac{F(r)}{r}=a, \quad 0<a \leqq \infty ;
$$

then corresponding to each pair of positive numbers $b, c$ satisfying the inequalities $b<a ; 1<c<a / b$, there is a sequence $R_{1}, R_{2}, \cdots$ tending to $\infty$ such that $R_{1}>r_{0}$ and

$$
F(r)>b r, \quad R_{n} \leqq r \leqq c R_{n}<R_{n+1} ; \quad n=1,2, \cdots .
$$

LEMMA 2 [5, pp. 4-10]. If $\nu(r)$ denotes the rank of the maximum term of $f(z)$ and $M_{j}(r)$ denotes the maximum modulus of $f^{(j)}(z)$, then for $j=1,2, \cdots$,

$$
(\nu(r) / r)^{j} M(r)\left(1-\epsilon_{j}(r)\right) \leqq M_{j}(r) \leqq(\nu(r) / r)^{j} M(r)\left(1+\epsilon_{j}(r)\right),
$$

where $0 \leqq \epsilon_{j}(r) \rightarrow 0$, for $r \rightarrow \infty$ outside a set of finite logarithmic measure.

Lemma 3 [3]. Let $f(z)$ be an entire function of positive order $\rho$. Write

$$
\limsup _{r \rightarrow \infty} \frac{\log M(r)}{r^{\rho}}=T, \quad \limsup _{r \rightarrow \infty} \frac{\nu(r)}{r^{\rho}}=\gamma .
$$

Then $\rho T \leqq \gamma \leqq e \rho T$.

Proof OF THEOREM. Let

$$
\limsup _{r \rightarrow \infty} \frac{\nu(r)}{r}=a \leqq \infty .
$$

We will show that

$$
a \leqq N+1 .
$$

We may suppose that $a>0$, for otherwise (7) is obviously true. We take $b$ and $c$ as in Lemma 1 and denote by $E_{n}$ the interval $\left[R_{n}, c R_{n}\right]$. Write $G=\cup_{1}^{\infty} E_{n}$. The variation of $\log r$ in $E_{n}$ is $\log c$, and so the total variation of $\log r$ over $\bigcup_{1}^{p} E_{n}$ tends to infinity with $p$. Let $E$ denote the set, on the $r$-axis, outside which (5) holds for $j=1,2, \cdots, N+1$. Hence the set $G \cap C E$, where $C E$ is the complement of the set $E$, contains a sequence of points $r_{n} \uparrow \infty$. Let $0<b_{1}<b$. Then for $r$ $=r_{n}\left(n>n_{0}\right)$,

$$
\begin{aligned}
M_{j}(r) / M_{p}(r) \sim(\nu(r) / r)^{j-p} \geqq b^{j-p} \\
\quad 0 \leqq p \leqq j, \quad j=1,2, \cdots, N+1 .
\end{aligned}
$$

Since $f(z)$ is of index $N$, we have 


$$
M_{N+1}(r) \leqq(N+1) ! \max \left\{M(r), M_{1}(r) / 1 !, \cdots, M_{N}(r) / N !\right\},
$$

and hence for $r=r_{n}, n>n_{1}$,

$M_{N+1}(r)$

$$
\leqq(N+1) ! \max \left\{M_{N}(r) / b_{1}^{N}, \cdots, M_{N}(r) /(N-1) ! b_{1}, M_{N}(r) / N !\right\} .
$$

If $b_{1} \leqq N$, then (7) is obviously true and so we may suppose that $b_{1}>N$. Then for $r=r_{n}$,

$$
M_{N+1}(r)<(N+1) ! M_{N}(r) / N ! \leqq(N+1) M_{N+1}(r) / b_{1},
$$

and so $b_{1} \leqq N+1$ and (7) follows. From (7) and Lemma 3 we deduce that $f(z)$ is of exponential type $T \leqq N+1$.

To show that (1) is sharp, we consider $f(z)=\exp ((N+1) z)$ where $N$ is a nonnegative integer. This function is of index $N$ and type $T=N+1$.

3. Proof of Theorem 2. We may suppose without loss of generality that $c<1$. Let

$$
\Psi(r)=\min \left\{2 \phi(r) / c, \exp \left(\log ^{2}(2+r)\right)\right\} .
$$

Write $F(r)=(\Psi(r))^{1 / 2}$ and let

$$
\eta(r)=1 / \sup _{t \geq r}[\log t / \log F(t)] .
$$

Then $\eta(r) \uparrow \infty$ and $\eta(r) \leqq \frac{1}{2} \log ^{2}(2+r) / \log r$. Let $r_{1} \geqq e$ be chosen such that $\eta\left(r_{1}\right) \geqq 1$ and write $m_{1}=\left[\eta\left(r_{1}\right)\right]$. Let $r_{n}(n=2,3, \cdots)$ be chosen such that $\eta\left(r_{n}\right) \geqq 2 \eta\left(r_{n-1}\right)$ and write $m_{n}=\left[\eta\left(r_{n}\right)\right]-\left[\eta\left(r_{n-1}\right)\right], \quad n=2$, $3, \cdots$. Let

$$
\xi(z)=\prod_{1}^{\infty}\left(1+\frac{z}{r_{n}}\right)^{m_{n}} .
$$

Then for $r_{n} \leqq r<r_{n+1}, n(r)=m_{1}+\cdots+m_{n}=\left[\eta\left(r_{n}\right)\right] \leqq \eta(r)=O(\log r)$. Hence $\xi(z)$ is an entire function and [1, pp. 47-49]

$$
\log \xi(r) \sim N(r)=\int_{e}^{r} \frac{n(t)}{t} d t \leqq n(r) \log r \leqq \eta(r) \log r \leqq \log F(r) .
$$

Hence for all $r \geqq r_{0}$,

$$
\log \xi(r) \leqq 2 \log F(r)=\log \Psi(r) .
$$

Let

$$
f(z)=c \xi(z) / 2 \xi\left(r_{0}\right) .
$$

Then $f(z)$ is an entire function such that for $r \geqq 0, M(r, f) \leqq \phi(r)$. 
Furthermore, $f(z)$ is of unbounded index for it has zeros of arbitrarily large order.

4. Proof of Theorem 3. For any integer $p \geqq n-1$, we have

$$
\begin{aligned}
\max \left\{|f|, \cdots, \frac{\left|f^{(p)}\right|}{p !}\right\} \geqq & \frac{\left|f^{(p)}\right|}{p !}\left(\frac{\left|a_{1}\right|}{p+1}\right) \\
& +\frac{\left|f^{(p-1)}\right|}{(p-1) !}\left(\frac{\left|a_{2}\right|}{p(p+1)}\right)+\cdots \\
& +\frac{\left|f^{(p-n+1)}\right|}{(p-n+1) !} \frac{\left|a_{n}\right|}{(p-n+2) \cdots(p+1)}
\end{aligned}
$$

provided

$$
\frac{\left|a_{1}\right|}{p+1}+\frac{\left|a_{2}\right|}{p(p+1)}+\cdots+\frac{\left|a_{n}\right|}{(p-n+2) \cdots(p+1)} \leqq 1,
$$

which is condition (3). Now by (2)

$$
f^{(p+1)}+a_{1} f^{(p)}+\cdots+a_{n} f^{(p-n+1)}=0,
$$

and hence

$$
\frac{\left|f^{(p+1)}\right|}{(p+1) !}=\frac{\left|a_{1} f^{(p)}+\cdots+a_{n} f^{(p-n+1)}\right|}{(p+1) !} .
$$

This proves that

$$
\max \left\{|f|, \cdots, \frac{\left|f^{(p)}\right|}{p !}\right\} \geqq \frac{\left|f^{(p+1)}\right|}{(p+1) !} .
$$

With the help of the equation (2), we can show that the inequality (10) holds, when $(p+1)$ in the expression on the right of (10) is replaced by $j$, where $j=p+2, p+3, \cdots$. Hence $N \leqq p$ and the theorem is proved. To prove the corollary, note that (4) implies $p \geqq n-1$ and

$$
p-n+2 \geqq \max \left(\left|a_{1}\right|,\left|a_{2}\right|^{1 / 2}, \cdots,\left|a_{n}\right|^{1 / n}\right) .
$$

Hence

$$
\begin{aligned}
& (p+1) p \cdots(p-n+2) \\
& \geqq(p+1) p \cdots(p-n+3)\left(\left|a_{1}\right|+\left|a_{2}\right|^{1 / 2}+\cdots+\left|a_{n}\right|^{1 / n}\right) \\
& \geqq p(p-1) \cdots(p-n+2)\left|a_{1}\right|+(p-1) \cdots(p-n+2)\left|a_{2}\right| \\
& \quad+\cdots+\left|a_{n}\right|,
\end{aligned}
$$

and so (3) is satisfied. 
5. Remarks and examples. (a) Let $f(z)=P(z) e^{a z}$ where $a$ is any complex number and $P(z)$ is a polynomial of degree less than $n$. Then $f(z)$ is of bounded index, and the index $N \leqq p$ where $p$ is any integer such that $p \geqq n-1$ and

$$
\begin{aligned}
\frac{n|a|}{p+1}+\left(\frac{n(n-1)}{2 !}|a|^{2}\right) \frac{1}{p(p+1)} & +\cdots \\
& +\frac{|a|^{n}}{(p-n+2) \cdots(p+1)} \leqq 1 .
\end{aligned}
$$

For $D^{n}\left\{f(z) e^{-a z}\right\}=0$ and so

$$
f^{(n)}+(-a n) f^{(n-1)}+\left\{a^{2} \frac{n(n-1)}{2 !}\right\} f^{(n-2)}+\cdots+(-1)^{n} a^{n} f=0 .
$$

Hence the result follows from Theorem 3.

(b) If an entire function $f(z)$ satisfies equation (3) and $c \neq 0$ is any constant, then $F(z)=f(c z)$ is of bounded index.

(c) If $f(z)$ is of bounded index, then index $f(z)=$ index $f(\alpha z)$ where $\alpha$ is a constant of modulus 1.

(d) Let $f(z)$ be an entire function satisfying the equation

$$
f^{(2)}+a_{1} f^{(1)}+a f=0 .
$$

If $F(z)=f(\sqrt{ } z)$ is an entire function, then $F(z)$ is of bounded index.

Since $f(\sqrt{ } z)$ is entire we must have $a_{1}=0$. We may suppose, without loss of generality, that $a>0$. Solving the equation and noting that $f(z)$ is an even function we get $F(z)=a_{0} \cos (a z)^{1 / 2}$. Since $F(z)$ and $c F(z)$ have the same index we need consider

$$
F(z)=\cos (a z)^{1 / 2}, \quad a>0 .
$$

The zeros of $F(z)$ are all real and $F(z)$ is real for real $z$. Hence by Laguerre's theorem $\left[1\right.$, p. 23] the zeros of $F^{1}(z)$ are all real and are separated from each other by the zeros of $F(z)$. We repeat this argument with $F^{(1)}(z), F^{(2)}(z), \cdots$ and deduce that if $\left\{z_{0, n}\right\}_{1}^{\infty},\left\{z_{1, n}\right\}_{1}^{\infty}, \cdots$ denote respectively the zeros of $F(z), F^{1}(z), \cdots$, then $0<z_{0,1}<z_{1,1}$ $<\ldots$. It is easy to see that $z_{k, 1} \rightarrow \infty$ with $k$. Hence we can choose smallest integer $k$ such that $k \geqq \max \left(a-\frac{1}{2}, 1\right)$ and

$$
F^{(k)}(z) \text { has no zeros in }|z|<1 \text {. }
$$

Then 


$$
\begin{aligned}
\min _{|z|=1} \mid & F^{(k)}(z) \mid \\
& =\min _{|z| \leqq 1}\left|F^{(k)}(z)\right| \\
& =\frac{k ! a^{k}}{(2 k) !}\left\{1-\frac{a(k+1)}{(2 k+1)(2 k+2)}+\frac{a^{2}(k+1)(k+2)}{(2 k+1) \cdots(2 k+4)}-\cdots\right\} \\
& \geqq \frac{2}{3} \frac{k ! a^{k}}{(2 k) !} .
\end{aligned}
$$

If $j \geqq k$,

$$
\max _{|z| \leqq 1}\left|F^{(j)}(z)\right| \leqq \frac{4}{3} \frac{j ! a^{j}}{(2 j) !} .
$$

Hence for $|z| \leqq 1$,

$$
\max \left\{|F|, \cdots,\left|F^{(k)}\right| / k !\right\} \geqq\left|F^{(j)}\right| / j !, \quad j=1,2, \cdots
$$

For $|z| \geqq 1$

$$
\left|F^{(k+1)}\right| /(k+1) !=\left|(4 k-2) F^{(k)}+a F^{(k-1)}\right| / 4\left|g_{z}\right|(k+1) ! .
$$

Now the left expression of (12)

$$
\geqq\left(\left|F^{(k)}\right| / k !\right) \alpha+\left(\left|F^{(k-1)}\right| /(k-1) !\right) \beta
$$

provided $0 \leqq \alpha, 0 \leqq \beta$ and $\alpha+\beta \leqq 1$. Take

$$
\alpha=(4 k-2) / 4|z|(k+1), \quad \beta=a / 4|z| k(k+1) .
$$

Then $\alpha+\beta \leqq 1$ and so (12) holds for $|z| \geqq 1$ and $j=k+1$. Similarly it holds for $j=k+2, k+3, \cdots$. Therefore $F(z)$ is of index $N \leqq k$.

\section{REFERENCES}

1. R. P. Boas, Jr., Entire functions, Academic Press, New York, 1954.

2. B. Lepson, Differential equations of infinite order, hyperdirichle tseries and entire functions of bounded index, Lecture notes, 1966, Summer Institute on Entire Functions, Univ. of California, La Jolla.

3. S. M. Shah, $A$ note on the derivatives of integral functions, Bull. Amer. Math. Soc. 53 (1947), 1156-1163.

4. - The maximum term of an entire series. III, Quart. J. Math. Oxford Ser. 19 (1948), 220-223.

5. Hans Wittich, Neuere Untersuchungen über eindeutige analytische Funktionen, Springer-Verlag, Berlin, 1955.

UNIVERSITY OF KENTUCKY 